\title{
Novel characterisation of mast cell phenotypes from peripheral blood mononuclear cells in chronic fatigue syndrome/myalgic encephalomyelitis patients
}

\author{
Thao Nguyen, ${ }^{1,2}$ Samantha Johnston, ${ }^{1,2}$ Anu Chacko, ${ }^{1,2}$ Damien Gibson, ${ }^{1,2}$ Julia Cepon, ${ }^{1,2}$ Peter Smith, ${ }^{2}$ Donald Staines, ${ }^{1,2}$ \\ Sonya Marshall-Gradisnik ${ }^{1,2}$
}

\begin{abstract}
Background: Mast cells (MCs) mediate inflammation through neuropeptides and cytokines, along with histamine and reactive oxygen species (ROS). Chronic Fatigue Syndrome/Myalgic Encephalomyelitis (CFS/ME) is an illness characterized by an unexplained disabling fatigue with multiple physiological impairments as well as dysregulated cytokine profiles.
\end{abstract}

Objective: To determine mast cell phenotypes in isolated human PBMCs, in healthy controls and in CFS/ME patients. Second, determine receptor expression of RAGE and its ligand high mobility group box 1 protein (HMGB1).

Method: Moderately severe CFS/ME patients ( $n=12$, mean age $39.25 \pm$ SD3.52 years), severe CFS/ME patients ( $n=6$, mean age $43.00 \pm$ SD4.02 years) and healthy controls $(n=13$, mean age $42.69 \pm$ SD3.87 years) were included in this study. CFS/ME patients were classified according to the 2011 International Consensus Criteria. LSRFortessa X-20 Flow cytometry was used for the identification of phenotypic peripheral mast cell population in PBMCs using an exclusion marker Lin2 cocktail (anti-CD3, anti-CD14, anti-CD19, anti-CD20 and anti-CD56) and inclusion markers (CD117, CD34, FCeRI, chymase, HLA-DR and CD154) following comparative investigation. HMGB1 and soluble RAGE expression in plasma was measured by sandwich ELISA assay.

Results: There was a significant increase in $\mathrm{CD} 117^{+} \mathrm{CD} 34^{+} \mathrm{FC} \varepsilon \mathrm{RI}^{-}$chymase ${ }^{-}$mast cell populations in moderate and severe CFS/ME patients compared with healthy controls. There was a significant increase in CD40 ligand and MHC-II receptors on differentiated mast cell populations in the severe CFS/ME compared with healthy controls and moderate CFS/ME. There were no significant differences between groups for HMGB1 and sRAGE.

Conclusions: This preliminary study investigates mast cell phenotypes from PBMCs in healthy controls. We report significant increase of naïve MCs in moderate and severe CFS/ME patients compared with healthy controls. Moreover, a significant increase in CD40 ligand and MHC-II receptors on differentiated mast cells in severe CFS/ME patients. Peripheral MCs may be present in CFS/ME pathology however, further investigation to determine their role is required.

Keywords: Chronic Fatigue Syndrome; Mast Cells; Receptor for advanced glycation end products; RAGE; Ligand High Mobility Group Box 1 protein (HMGB1); Myalgic Encephalomyelitis

\section{From:}

${ }^{1}$ School of Medical Science, Griffith University, Gold Coast, QLD, Australia

2 The National Centre for Neuroimmunology and Emerging Diseases, Menzies Health Institute Queensland, Griffith University, Gold Coast, QLD Australia

Corresponding author:

Thao Nguyen

Griffith University, Menzies Health Institute Queensland,

National Centre for Neuroimmunology and Emerging Diseases,

Southport, QLD 4222, Australia

Email: thao.nguyen@griffith.edu.au

\section{Introduction}

Mast cells (MCs) are found in the blood circulating as an immature form, prior to migrating to vascularised tissues, where they differentiate into functional cells such as connective tissue (or serosal) and mucosal MCs. ${ }^{1}$ Importantly, mature cells do not ordinarily circulate in peripheral blood ${ }^{2}$ as they are released as immature cells into the blood periphery where they migrate throughout the body to tissues such as the skin, mucosa, brain and airways to undergo maturation. ${ }^{3}$ Stem-cell factor, also known as c-kit, is the ligand for the receptor responsible for the main survival and developmental factor 
for MCs. Additionally, immune modulators, such as growth factors, cytokines and chemokines are able to affect MCs and their phenotypes. ${ }^{2,4,5} \mathrm{MC}$ phenotypes that have been well recognised are defined according to their protease content. ${ }^{6}$ MC activation and degranulation can occur from FCeRI (IgE cross-linking) or independent mechanisms such as, other immunoglobulin receptor $(\mathrm{FC} \gamma \mathrm{R})$, cytokines, chemokines, neuropeptides and through receptors involved in mast cell interaction with surrounding immune cells (MHC-II, CD40L, OX40). MC phenotype is determined by the level of surface expression of the key differentiating CD markers such as CD117 (c-kit), FCeRI and CD33, where bright and dim populations can been seen, along with the surface expression of CD34. The CD34 maker is expressed during the immature stages of the MC. ${ }^{7,8}$ Mast cells that are differentiated, mature or activated, express FCeRI and HLA-DR. ${ }^{9}$ Activated MCs produce and release several pro-inflammatory mediators from intracellular stores which alter the inflammatory environment to mobilise immune cells to the site of the pathogen infection and to draining lymph nodes. ${ }^{2}$ Importantly, human MC phenotypes have been characterised in bone marrow and tissues from pathological diseases. They have not been well characterised from peripheral blood.

Previous investigations have characterised MC phenotypes from bone narrow and tissue in pathological diseases using predominate methods that are poorly reproducible, expensive to produce, biological properties differ from in vivo i.e human primary cell culture model or primary cell lines. Thus, there is a need for development of a relevant, reproducible and inexpensive in vitro model to represent $\mathrm{MC}$ found in vivo morphological and biological properties. Therefore, identification of MC from peripheral blood using flow cytometric methods, facilitate and elucidate MC phenotype and function in healthy and diseases.

Chronic Fatigue Syndrome/Myalgic Encephalomyelitis (CFS/ME) is a debilitating heterogeneous disorder associated immune, nerological, musculoskeletal, gastrointestinal and autonomic symptoms. The aetiology of CFS/ME is unknown, however, immune dysfunction has been consistently implicated in the pathomechanism of the illness. ${ }^{10}$ Atypical levels of regulatory $\mathrm{T}$ cells (Treg) and pro-inflammatory-and anti-inflammatory cytokines, generation of nitric oxide and hypersensitivity responses suggest that the pathophysiology of CFS/ME involves the activation of inflammatory pathways. ${ }^{6,11-13}$ Nuclear factor kappa beta $(\mathrm{NF}-\kappa \mathrm{B})$ is a transcriptional factor that plays a role in inflammatory pathways, cell proliferation, differentiation, and survival. Previous investigators have reported significant increases in NF- $\kappa \mathrm{B}$ in CFS/ME patients. ${ }^{14}$

The Receptor for Advanced Glycation End products (RAGE) is a multi-ligand member of the immunoglobulin superfamily of cell surface molecules. ${ }^{15,16}$ Its ligands include the high mobility group box 1 protein (HMGB1) and AGEs, which are known to play a major role in the amplification of chronic inflammation and are associated with inflammation. ${ }^{17,18}$ Hence it was important to know if self-perpetuating inflammatory signals were being promulgated by mast cells activated by RAGE in CFS/ME.
The purpose of this investigation was to identify MC phenotypes in isolated human PBMCs using flow cytometry and the soluble receptor for RAGE, as well as its ligand high mobility group box 1 protein (HMGB1), in healthy controls and CFS/ME patients.

\section{Methods \\ Subjects}

CFS/ME patients were aged matched with healthy controls (Table 1). Thirteen healthy controls (aged $42.69 \pm 3.87$ years), twelve moderate CFS/ME (aged $39.25 \pm 3.52$ ) and six severe CFS/ME patients (aged $43.00 \pm 4.03$ ) participated in this study. CFS/ME patients were defined in accordance with the International Consensus Criteria ${ }^{10}$ and excluded if they had any disease that would exclude CFS/ME diagnosis, were smokers, pregnant or breast feeding. Severity of fatigue and grouping of CFS/ME patients was determined using Dr David Bell's disability scale (DBDS) ${ }^{19}$ Moderate CFS/ME were classified as patients scoring $30 \%$ or higher on the Dr Bell's Disability Score (DBDS). Severe CFS/ME were classified as patients scoring less than $30 \%$ on the DBDS and according to this scale, are considered housebound or bedridden. All healthy control participants did not meet CFS/ME criteria. All participants were of Caucasian decent and residents of Australia at the time of blood collection. Participants gave written consent prior to blood collection and this study was conducted under the approval of Griffith University Ethics Reference number (MSC/23/12/HREC).

$30 \mathrm{~mL}$ of whole blood was collected from all participants where $10 \mathrm{~mL}$ of whole blood was collected into EDTA tubes to investigate full blood count, electrolytes, high sensitive $\mathrm{C}$ reactive protein and erythrocyte sedimentation rate. There were no significant differences between groups for these blood parameters (Table 1).

$10 \mathrm{ml}$ EDTA whole blood was collected for mast cell phenotypic immune assay and $8 \mathrm{ml}$ of CPT sodium citrate (BD Bioscience, Franklin Lakes, NJ) for HMGB1 and sRAGE ELISA assays.

\section{Mast cell phenotypic immune assay}

Peripheral blood mononuclear cells (PBMCs) isolation was performed using Ficoll-Paque PLUS density centrifugation (GE healthcare, Parramatta NSW) to help remove granulocytes (such as, neutrophils, basophils and eosinophils). PBMCs were stained with trypan blue stain (Invitrogen, Carlsbad, CA) for cell viability count and adjusted in a final concentration of $1 \times 10^{7}$ cells in $150 \mu \mathrm{L}$. Cells were labelled with the mononuclear antibodies for 30 minutes, excepted CD34 were incubated for 45 minutes. Intracellular staining of chymase was performed with $\mathrm{BD}$ fix and perm per manufacture's instruction (BD Bioscience, San Jose, CA). Labelled cells were resuspended with stained buffer (BD Bioscience, San Jose, CA) prior to flow cytometric analyses. Isotypes was used for negative control (Table 1).

\section{Flow cytometry}

LSR Fortessa ${ }^{\mathrm{TM}} \mathrm{X}-20$ flow cytometry was employed to analyse mast cell phenotype. All samples were collected 
Table 1. Participant demographics and pathology

\begin{tabular}{|c|c|c|c|c|}
\hline & Healthy controls $(n=13)$ & Moderate CFS/ME (n=12) & Severe CFS/ME $(n=6)$ & P Value \\
\hline Age (years) & $42.69 \pm 3.87$ & $39.25 \pm 3.52$ & $43.00 \pm 4.03$ & 0.469 \\
\hline Sex (\% female) & $61.50 \%$ & $91.70 \%$ & $50 \%$ & 0.038 \\
\hline Dr Bell's Score (\%) & $93.08 \pm 7.48$ & $52.50 \pm 7.23$ & $25.00 \pm 13.17$ & 0.000 \\
\hline \multicolumn{5}{|l|}{ Pathology } \\
\hline White Cell Count $($ x 10^9/L) & $6.48 \pm 0.82$ & $6.25 \pm 0.59$ & $6.02 \pm 0.51$ & 0.962 \\
\hline Lymphocytes $($ x 10^9/L) & $1.99 \pm 0.171$ & $1.82 \pm 0.16$ & $2.01 \pm 0.25$ & 0.745 \\
\hline Monocytes $($ x 10^9/L) & $0.31 \pm 0.03$ & $0.34 \pm 0.02$ & $0.36 \pm 0.04$ & 0.557 \\
\hline Neutrophils (x 10^9/L) & $4.04 \pm 0.728$ & $3.91 \pm 0.49$ & $3.40 \pm 0.29$ & 0.877 \\
\hline Eosinophils (x 10^9/L) & $0.13 \pm 0.03$ & $0.16 \pm 0.03$ & $0.23 \pm 0.08$ & 0.252 \\
\hline Basophils (x 10^9/L) & $0.02 \pm 0.00$ & $0.03 \pm 0.00$ & $0.03 \pm 0.01$ & 0.905 \\
\hline Platelets $($ x 10^9/L) & $240.31 \pm 15.61$ & $278.67 \pm 19.71$ & $230.17 \pm 20.66$ & 0.144 \\
\hline Haemoglobin $(\mathrm{g} / \mathrm{L})$ & $140.69 \pm 3.17$ & $135.42 \pm 2.26$ & $135.83 \pm 3.76$ & 0.242 \\
\hline Haematocrit & $0.42 \pm 0.01$ & $0.41 \pm 0.01$ & $0.41 \pm 0.01$ & 0.449 \\
\hline Red Cell Count $\left(\mathrm{x} 10^{\wedge} 12 / \mathrm{L}\right)$ & $4.82 \pm 0.01$ & $4.52 \pm 0.06$ & $4.55 \pm 0.15$ & 0.300 \\
\hline Mean Corpuscular Volume (fL) & $87.38 \pm 1.66$ & $89.75 \pm 0.70$ & $90.33 \pm 1.84$ & 0.316 \\
\hline Sodium $(\mathrm{mmol} / \mathrm{L})$ & $138.08 \pm 0.57$ & $137.25 \pm 0.40$ & $138.33 \pm 0.49$ & 0.275 \\
\hline Potassium (mmol/L) & $4.16 \pm 0.09$ & $4.11 \pm 0.08$ & $3.83 \pm 0.17$ & 0.208 \\
\hline Chloride $(\mathrm{mmol} / \mathrm{L})$ & $102.54 \pm 0.43$ & $102.25 \pm 0.46$ & $102.50 \pm 1.34$ & 0.889 \\
\hline Bicarbonate (mmol/L) & $27.31 \pm 0.59$ & $27.42 \pm 0.50$ & $27.83 \pm 1.47$ & 0.806 \\
\hline Anion Gap (mmol/L) & $8.23 \pm 0.30$ & $7.50 \pm 0.34$ & $7.83 \pm 0.60$ & 0.324 \\
\hline Erythrocyte sedimentation rate $(\mathrm{mm} / \mathrm{Hr})$ & $11.31 \pm 2.41$ & $15.50 \pm 3.47$ & $10.50 \pm 2.61$ & 0.218 \\
\hline High sensitive C-Reactive Protein & $2.635 \pm 0.83$ & $4.08 \pm 1.65$ & $0.50 \pm 0.22$ & 0.174 \\
\hline
\end{tabular}

${ }^{*}$ means significance, $P<0.05$

at 200,000 events. All antibodies were purchase from BD Bioscience, unless otherwise stated. Cells exclusion markers were Lin2 cocktail (anti-CD3, anti-CD14, anti-CD19, anti-CD20 and anti-CD56) to exclude lymphocytes, monocytes, eosinophil and neutrophils and inclusion markers and protein to help identify mast cells were CD117, CD34, FCeRI, and chymase. Mast cells were further characterised by interaction markers HLA-DR (MHC-II), CD154 (CD40L). ${ }^{20-22}$ Mast cells subsets were defined as mast cell progenitor $\left(\mathrm{CD} 117^{+} \mathrm{CD} 34^{+}\right.$ FCeRI-chymase $\left.{ }^{-}\right),{ }^{9,23,24}$ mature mast cell $\left(\mathrm{CD} 117^{+} \mathrm{CD} 34^{-} \mathrm{FC} \varepsilon \mathrm{RI}^{-}\right)$ activated mast cell $\left(\mathrm{CD} 117^{+} \mathrm{CD} 34-\mathrm{FC} \varepsilon \mathrm{RI}-\mathrm{HLA}^{-} \mathrm{DR}^{+} \mathrm{CD} 40 \mathrm{~L}^{+} .{ }^{25}\right.$

\section{HMGB1 and sRAGE sandwich ELISA}

Sodium citrate blood tubes were centrifuge at $1800 \mathrm{~g}$ for 20 minutes at $20^{\circ} \mathrm{C}$ to collect $3 \mathrm{ml}$ of plasma. Plasma was stored at $-20^{\circ} \mathrm{C}$ until analysed. Sandwich ELISA assay for HMGB1 (IBL International GMBH, Hamburg, Germany) and sRAGE (R\&D Systems, Minneapolis, USA) was performed as per manufacturer's instructions. Briefly, 50 $\mathrm{ll}$ of standards and plasma from healthy controls, moderate and severe CFS/ ME groups were plated in duplicate on a 96 microtiter plate that was pre-coated with either human anti-sRAGE or anti-HMGB1 monoclonal antibody. Prior to labelling HMGB1 and sRAGE with anti-HMGB1, 2 conjugated with peroxidase or anti-sRAGE conjugated with horseradish peroxidase, the plate was washed with manufacturer's buffer using an automatic plate washer. Reactions were stopped with $100 \mu \mathrm{l}$ of sulfuric acid prior to measuring the optical density with POLARstar Omegaversion OMEGA 1.20. The optical density was measured at $450 \mathrm{~nm}$, with wavelength correction set to $540 \mathrm{~nm}$ and $600 \mathrm{~nm}$ for sRAGE and HMGB1 plate, respectively. The average $\mathrm{OD}$ for each sample was determined, and was corrected for the OD of the blank well. Linear standard curves were analysed to determine samples sRAGE and HMGB1 concentration (Figure 1)

\section{Statistical analyses}

Data was analysed using SPSS Version 22. Shapiro-wilk normality test was used to determine the distribution of data. Demographics of participants was normally distributed and ANOVA was used to test for significance. Kruskal-Wallis test was performed to determine the statistical significance of pathology test, mast cell phenotype, HMGB1 and soluble RAGE between groups. Mann-Whitney U tests determined the significance between groups for both non-parametric and parametric data and reported as $\mathrm{p}<0.05$.

\section{Results}

There were no significant differences between groups for gender, age and pathology results (Table 1). There was a significant difference between groups for the Dr Bell's Fatigue Severity Score where the mean score for the healthy control group was $92 \%$ compared to the mean score for the moderate and severe CFS/ME groups at 53\% and 25\%, respectively (Table 1).

\section{Identification of human mast cells in peripheral blood}

Lin2 monoclonal antibody cocktail (anti-CD3, anti-CD14, anti-CD19, anti-CD20 and anti-CD56) was used to labelled immune cells (such as, T cells, B cells, natural killer cells, monocytes, eosinophil and neutrophils) is represented in black of the contour plot (Figure 1A). Cells labelled with a myeloid receptor, anti-CD117 were gated as $\mathrm{CD} 117^{+}$committed mast cells propagator (Figure 1A). CD $117^{+}$mast cells were further labelled with anti-CD34 and anti-FCeRI to differentiate four 
different phenotypes, including $\mathrm{FC} \mathrm{RI}^{+} \mathrm{CD} 34^{-}(\mathrm{Q} 1), \mathrm{FC} \mathrm{RI}^{+}$ $\mathrm{CD}^{+} 4^{+}(\mathrm{Q} 2)$, FCeRI- CD34- (Q3), and FCeRI- CD34+ (Q4). The four phenotypes also showed low and bright expression for intracellular chymase (Figure 1B) and interaction receptors CD40L and major histocompatibility complex class II (MHC-II) (Figure 1C). FCeRI+ CD34- represented in green (Q1) demonstrated higher expression of CD40L and MHC-II. Whereas $\mathrm{FC}_{2} \mathrm{RI}^{+} \mathrm{CD} 34^{+}(\mathrm{Q} 3)$ shown in blue and FCeRI- CD34 ${ }^{+}$ shown in orange (Q4) population demonstrated low/dim expression of MHC-II receptors and negative expression for
CD40L. Both MHC-II and CD40L receptors showed negative expression for $\mathrm{FCeRI}^{-} \mathrm{CD}^{-}$(Q3) displayed as the pink population (Figure 1C).

Increased levels of mast cell committed progenitors in moderate and severe CFS/ME patients

Mast cells expressing $\mathrm{CD} 117^{+} \mathrm{CD} 34^{+} \mathrm{FC} \varepsilon \mathrm{RI}^{-}$that did not express intracellular chymase were compared between the control, moderate and severe CFS/ME patients. CD $117^{+}$ $\mathrm{CD}^{2} 4^{+} \mathrm{FC} \varepsilon \mathrm{RI}^{-}$chymase ${ }^{-}$cells were significantly elevated in both
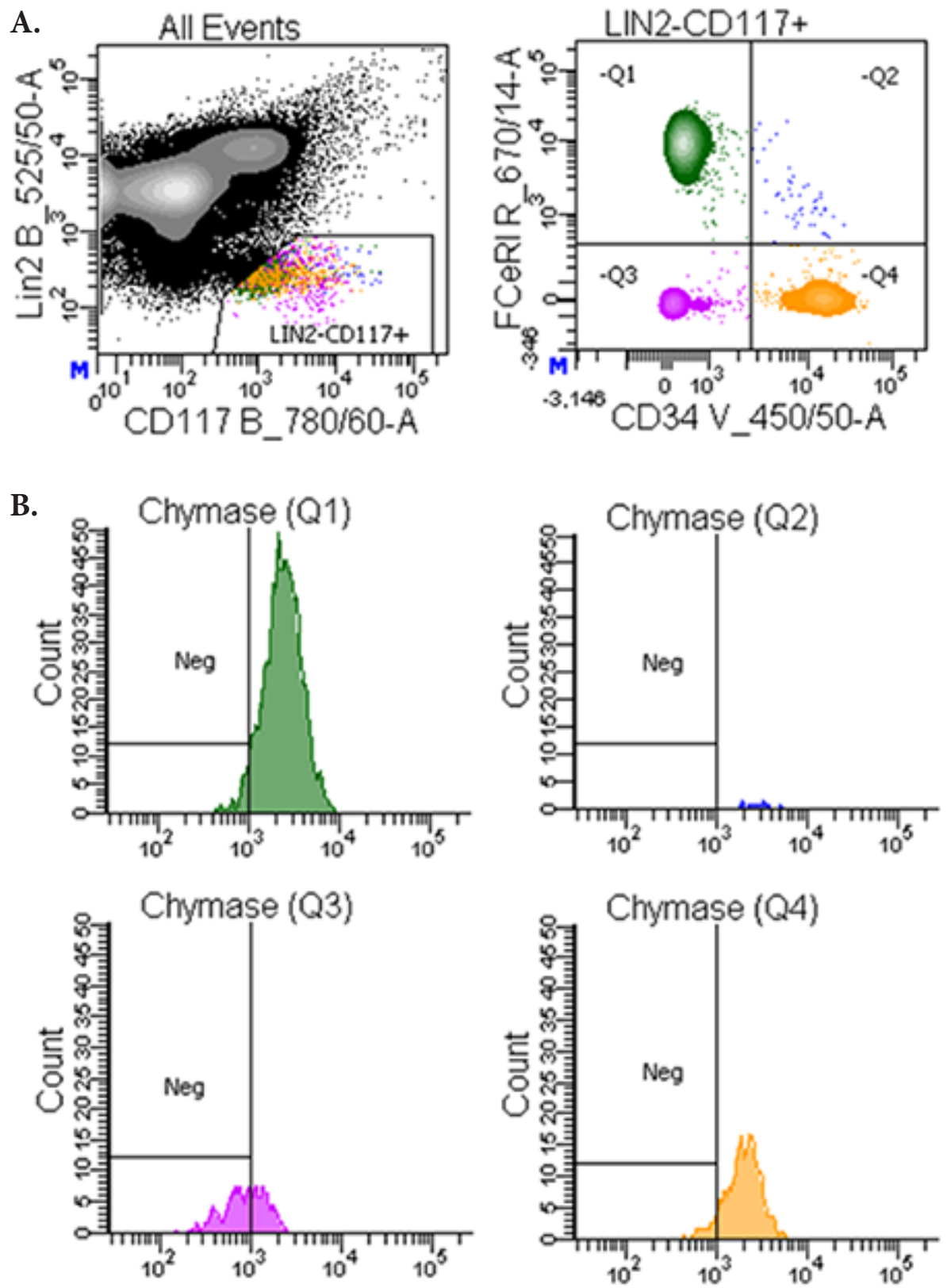

C.

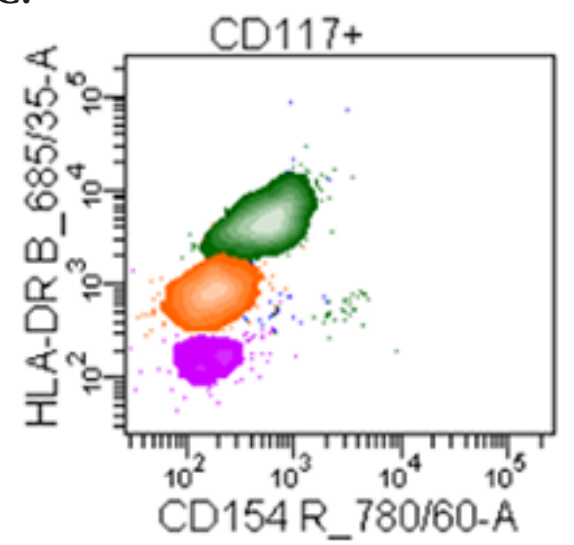

Figure 1. Identification and flow cytometry gating of human mast cells in peripheral blood. A. All PBMC events were plotted with Lin2 (anti-CD3, anti-CD14, anti-CD19, anti-CD20, anti-CD56) and CD117 antibodies to identify CD117 ${ }^{+}$cells. CD117 $7^{+}$cells were then gated for FCeRI and CD34 and the four phenotypes FCeRI ${ }^{+}$CD34- (Q1), FCeRI+ CD34+ (Q2), FCeRI- CD34- (Q3), and FCeRI- CD34+ (Q4) are represented. B. CD117 ${ }^{+}$cells subsets expressed differential levels of intracellular chymase. C. CD117 ${ }^{+}$cells subsets showed differential expression of HLA-DR and CD154 depending on the FCeRI and CD34 phenotype. HLA-DR is highly

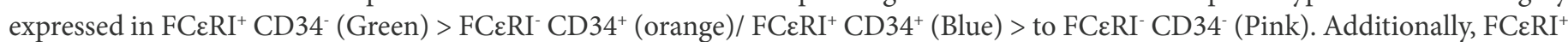
CD34- (Green) showed high expression for CD154 receptor. 
the moderate and severe CFS/ME patients compared with the healthy control group (Figure 2). No difference was observed between further subsets (Figure 1).

Upregulation of peripheral mast cell interaction receptors in severely affected CFS/ME patients

Surface markers CD154 and HLA DR receptors were compared between controls, moderate and severe CFS/ME patients. There was a significant increase in CD154 (CD40L) and HLA DR (MHC-II) receptors for the severe CFS/ME group compared to the healthy control and moderate CFS/ME group (Figure 3).

\section{sRAGE and its ligand HMGB1 in CFS/ME patients}

There was no significant difference between groups for HMGB1 and sRAGE (Figure 4).

\section{Discussion}

The present paper reports, for the first time, the identification of MC phenotypes in PBMCs from healthy individuals participating as controls. Flow cytometric methods

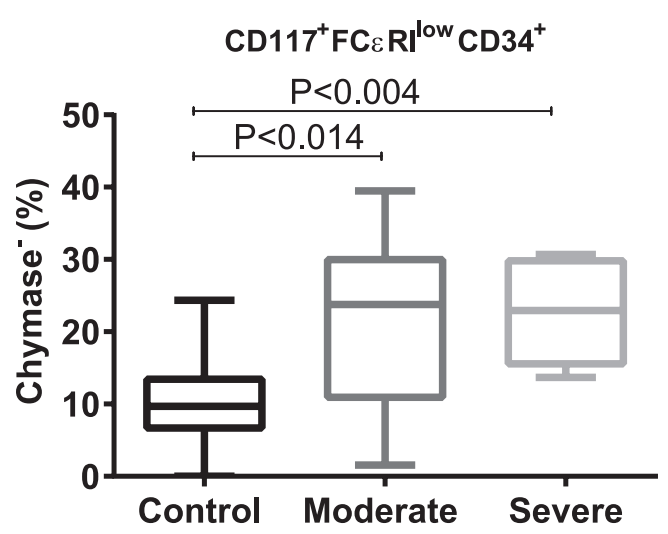

Figure 2. Box-whisker plot for mast cells in control, moderate and severe $\mathrm{CFS} / \mathrm{ME}$ patients. $\mathrm{CD} 117^{+} \mathrm{CD} 34^{+} \mathrm{FC} \varepsilon \mathrm{RI}^{-}$chymase mast cells are represented as percentage. The horizontal line within the box represents as medium and the outer horizontal lines of the box represents $25 \%$ and $75 \%$ percentile. The error bars denotes the minimum and maximum range. consisting of a cocktail of inclusion and exclusion cell markers, along with cell surface markers CD154 and MHC II (HLA-DR), were used for the first time. We were able to identify $\mathrm{MC}$ progenitor $\left(\mathrm{CD} 117^{+} \mathrm{CD} 34^{+} \mathrm{FC} \varepsilon \mathrm{RI}^{-}\right.$chymase),

A.

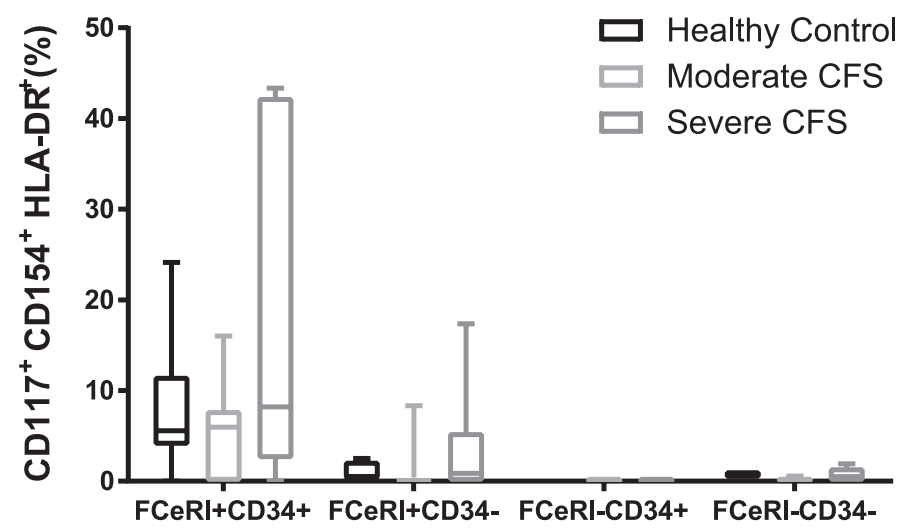

B.

$\mathrm{CD}_{117^{+} \mathrm{CD} 34^{-} \mathrm{FC}} \varepsilon \mathrm{RI}^{-}$

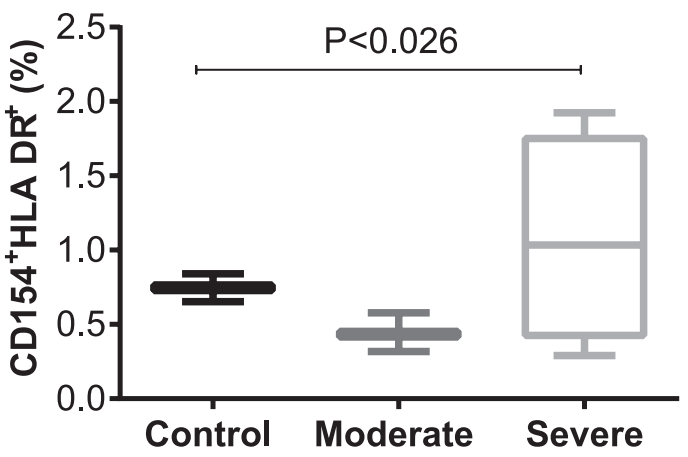

Figure 3. Immune profile of interaction markers expressed on mast cell surface. Box-whisker plots for CD154 $4^{+} \mathrm{HLA}-\mathrm{DR}^{+}$ is shown as percentage of parent $\mathrm{CD} 117^{+} \mathrm{CD} 344^{-\mathrm{FCRI}^{-}}$cells in controls, moderate and severe CFS/ME groups. The horizontal line within the box is represented as medium and the outer horizontal lines of the box are represented as $25 \%$ and $75 \%$ percentile. The error bars denotes the minimum and maximum range.

B.

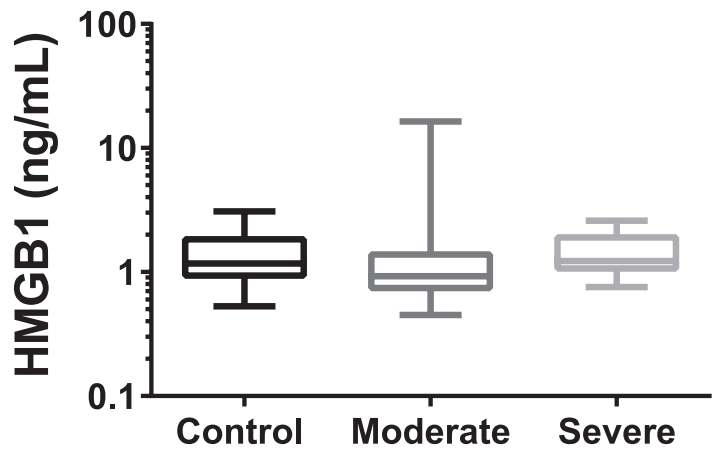

Figure 4. Comparison of plasma soluble RAGE (sRAGE) and HMGB1 in control, moderate and severe CFS/ME groups. A. sRAGE concentration was measured as $\mathrm{pg} / \mathrm{ml}$ between different severity groups. B. HMBG1 concentration was measured as ng/ml between different severity groups. 
mature $\mathrm{MCs}\left(\mathrm{CD} 117^{+} \mathrm{CD} 34{ }^{-} \mathrm{FCeRI}^{-}\right)$and activated $\mathrm{MCs}$ $\left(\mathrm{CD} 117^{+} \mathrm{CD} 34-\mathrm{FC} \varepsilon \mathrm{RI}-\mathrm{HLA}-\mathrm{DR}^{+} \mathrm{CD}^{-} \mathrm{LL}^{+}\right)$in human PBMCs from healthy individuals. In the comparative investigation we also report novel findings of a significant increase in CD $117^{+}$ $\mathrm{CD} 34^{+} \mathrm{FC} \varepsilon \mathrm{RI}^{-}$chymase ${ }^{-} \mathrm{MC}$ populations in moderate and severe $\mathrm{CFS} / \mathrm{ME}$ patients compared with healthy controls. Also we are the first to report a significant increase CD40 ligand and MHC-II receptors on differentiated MC populations for severe CFS/ME patients compared with the healthy control group and moderate CFS/ME patients.

A particular strength of this study is that it supports the identification of MC phenotypes and receptor expression in isolated human PBMCs using flow cytometric techniques without the need for morphological and cell culture based characterization.

Our research findings report a significant increase in mast cell progenitor (MCPs) $\left(\mathrm{CD} 117^{+} \mathrm{CD} 34^{+} \mathrm{FC} \varepsilon \mathrm{RI}^{-}\right.$chymase $\left.{ }^{-}\right)$in both moderate and severe CFS/ME patients compared with controls. MCPs circulate in the blood and lymphatics and migrate to peripheral tissues upon inflammation. ${ }^{26}$ MCPs have extensive proliferation potential and can differentiate into both connective and mucosal mast cells depending on the factors present in the tissue microenvironment. Mature mast cells are derived from MCPs under the influence of stem cell factor (SCF) secreted locally by fibroblasts, stromal cells and endothelial cells. ${ }^{27}$ Mast cell maturation and biological expression in the tissue microenvironment is influenced by cytokines specifically, IL-4, IL-10, IL-17, TNF- $\alpha$, and IFN- $\gamma$, which has been reported to be elevated in CFS/ME patients, suggesting that these cytokines may influence the tissue microenvironment and consequently the proliferation and differentiation of MCPs into mature mast cells in CFS/ME patients. ${ }^{28,29}$

Mast cells express a broad array of cell surface receptors and ligands which mediate the communication with immune cells belonging to both innate and acquired immunity, as well as with non-immune tissue-specific cell types. ${ }^{21}$ Our novel discovery of significant increase in CD154 (CD40L) and HLA DR (MHC-II) receptors for the severe CFS/ME group compared with the healthy control and moderate CFS/ ME group suggests MCs, macrophages as well as glial cells expressing the $\mathrm{CD} 40 \mathrm{~L}^{30}$ potentially may play a role in mediating immune dysregulation of $\mathrm{B}$ and $\mathrm{T}$ cell responses as well as contributing to neuroinflammation in CFS/ME patients. $^{21}$ MHC-II expression on MC effectively supports $\mathrm{T}$ cell proliferation and effector functions, subsequently leading to expansion of Tregs ${ }^{21,31}$ as well as B cell proliferation. Importantly, previous investigators have reported increased Treg cell numbers, B cell phenotypes and inflammation in CFS/ME patients. ${ }^{32}$

RAGE is known to play a role in MC activation, having been identified on both serosal and mucosal mast cells. ${ }^{33}$ Mechanisms proposed in RAGE activation of MC include reactive oxygen species production (ROS) and histamine release that may be involved in sensitivity, allergic reactions and immune-inflammation. Multiple chemical sensitivity for example, is a co-morbidity of CFS/ME. ${ }^{10}$ Secretory RAGE and its ligand, HMGB1, was not significantly different between groups. Further investigation into RAGE receptor expressed on
MC may provide understanding in MC inflammatory response in CFS/ME patients.

Mast cell activation has been associated with blood brain barrier (BBB) dysfunction such as neuroinflammation. Neuroinflammation on PET scans has been assessed in CFS/ ME patients. ${ }^{6}$ During neuroinflammation, mast cells can respond to neuropeptides, such as vasoactive intestinal polypeptide (VIP) in an FCeRI independent manner for degranulation and chemokine production. ${ }^{34}$ We have reported increased in VIP receptor (VPAC2) on CD4+ T cells and decreased IL-10 in cerebrospinal fluid from CFS/ME patients. ${ }^{35,36}$ Further, compared with controls, CFS/ME patients had significantly increased serum IFN-y, IL-5 and IL-10 compared with controls. ${ }^{37}$ Elevation in IFN-y is noteworthy, being a strong cytokine for antigen presentation and expression of MHC-II on mast cells. Detection of peripheral MC may possibly elucidate further pathways of neuroinflammation in the brain of CFS/ME patients.

\section{Conclusion}

We have discovered activated mast cells, for the first time, in CFS/ME patients as well as identifying mast cell phenotypes in blood of healthy controls. Moreover, the putative distribution of activated mast cells in almost all tissues and organs in the body requires further investigation to establish whether they have a role in the pathomechanism in CFS/ME. Importantly, neuroinflammatory processes and their sequelae involving the blood brain barrier and brain parenchyma should be investigated further in these patients.

\section{Author's contributions}

- SMG, TN, DRS designed and developed all experiments.

- TN, DG, JC, AC, PS and SJ assisted with the analysis of the data.

- SMG, DRS, SJ, and TN assisted with the preparation and final manuscript.

- SMG, DRS, PS, SJ, AC and TN assisted in the revisions of this manuscript.

\section{Acknowledgements}

This study was supported by funding from the Stafford Fox Medical Research Foundation, the Alison Hunter Memorial Foundation, Mason Foundation and Queensland Co-Investment Program.

\section{References}

1. Fukuda K, Straus SE, Hickie I, Sharpe MC, Dobbins JG, Komaroff A. The chronic fatigue syndrome: a comprehensive approach to its definition and study. International Chronic Fatigue Syndrome Study Group. Ann Intern Med. 1994;121:953-9.

2. Charcot J. Lectures on the diseases of the nervous system. New Sydenham Society. 1877.

3. McAlpine D, Compston, N. Some aspects of the natural history of disseminated sclerosis. Q J Med. 1952;21:135-67.

4. Brenu EW, van Driel ML, Staines DR, Ashton KJ, Hardcastle SL, Keane J, et al. Longitudinal investigation of natural killer cells and cytokines in chronic fatigue syndrome/myalgic encephalomyelitis. J Transl Med Epidemiol. 2012;10:88. 
5. Brenu EW, Huth TK, Hardcastle SL, Fuller K, Kaur M, Johnston S, et al. Role of adaptive and innate immune cells in chronic fatigue syndrome/ myalgic encephalomyelitis. Int Immunol. 2014;26:233-42.

6. Curriu M, Carrillo J, Massanella M, Rigau J, Alegre J, Puig J, et al. Screening NK-, B- and T-cell phenotype and function in patients suffering from Chronic Fatigue Syndrome. J Transl Med. 2013;11:68.

7. Lim ET, Giovannoni G. Immunopathogenesis and immunotherapeutic approaches in multiple sclerosis. Expert Rev Neurother. 2005;5:379-90.

8. Maghazachi AA. Role of Natural Killer Cells in Multiple Sclerosis. ISRN. 2012;2012:14.

9. Viglietta V, Baecher-Allan C, Weiner HL, Hafler DA. Loss of functional suppression by $\mathrm{CD} 4+\mathrm{CD} 25+$ regulatory $\mathrm{T}$ cells in patients with multiple sclerosis.J Exp Med. 2004;199:971-9.

10. Vantourout P, Hayday A. Six-of-the-best: unique contributions of gammadelta T cells to immunology. Nat Rev Immunol. 2013;13:88-100.

11. O'Keeffe J, Gately CM, Counihan T, Hennessy M, Leahy T, Moran AP, et al. T-cells expressing natural killer (NK) receptors are altered in multiple sclerosis and responses to alpha-galactosylceramide are impaired. J Neurol Sci. 2008;275:22-8.

12. Hardcastle SL, Brenu EW, Johnston S, Nguyen T, Huth T, Kaur M, et al. Analysis of the Relationship between Immune Dysfunction and Symptom Severity in Patients with Chronic Fatigue Syndrome/Myalgic Encephalomyelitis (CFS/ME). J Clin Cell Immunol. 2014;5:1-9.

13. Polman CH, Reingold SC, Banwell B, Clanet M, Cohen JA, Filippi M, et al. Diagnostic criteria for multiple sclerosis: 2010 revisions to the McDonald criteria. Ann Neurol. 2011;69:292-302.

14. Bell D. The Doctor's Guide to Chronic Fatigue Syndrome, Reading, Mass. Addison-Wesley Publishing Company, Reading, Mass 1995.

15. Anane LH, Edwards KM, Burns VE, Zanten JJ, Drayson MT, Bosch JA. Phenotypic characterization of gammadelta $\mathrm{T}$ cells mobilized in response to acute psychological stress. Brain Behav Immun. 2010;24(4):608-14

16. Venken K, Hellings N, Hensen K, Rummens JL, Medaer R, D’Hooghe M $\mathrm{B}$, et al. Secondary progressive in contrast to relapsing-remitting multiple sclerosis patients show a normal $\mathrm{CD} 4+\mathrm{CD} 25+$ regulatory T-cell function and FOXP3 expression. J Neurosci Res. 2006;83:1432-46.

17. Saresella M, Marventano I, Longhi R, Lissoni F, Trabattoni D, Mendozzi $\mathrm{L}$, et al. CD4+CD25+FOXP3+PD1-naive regulatory $\mathrm{T}$ cells in acute and stable relapsing-remitting multiple sclerosis and their modulation by therapy. FASEB J. 2008;22:3500-8.

18. Tivol EA, Borriello F, Schweitzer AN, Lynch WP, Bluestone JA, Sharpe AH. Loss of CTLA-4 leads to massive lymphoproliferation and fatal multiorgan tissue destruction, revealing a critical negative regulatory role of CTLA-4. Immunity. 1995;3:541-7.

19. Steiner K, Moosig F, Csernok E, Selleng K, Gross WL, Fleischer B, et al. Increased expression of CTLA-4 (CD152) by $\mathrm{T}$ and B lymphocytes in Wegener's granulomatosis. J Clin Exp Immunol. 2001;126:143-50.

20. Patsopoulos NA, Bayer Pharma MSGWG, Steering Committees of Studies Evaluating I-b, a CCRA, Consortium AN, GeneMsa, et al. Genome-wide meta-analysis identifies novel multiple sclerosis susceptibility loci. Ann Neurol. 2011;70:897-912.

21. International Multiple Sclerosis Genetics C, Hafler DA, Compston A, Sawcer S, Lander ES, Daly MJ, et al. Risk alleles for multiple sclerosis identified by a genomewide study. N Engl J Med. 2007;357:851-62.

22. Sellebjerg F, Krakauer M, Khademi M, Olsson T, Sorensen PS. FOXP3, CBLB and ITCH gene expression and cytotoxic T lymphocyte antigen 4 expression on CD4(+) CD25(high) T cells in multiple sclerosis. J Clin Exp Immunol. 2012;170:149-55.
23. Hammond KJ, Kronenberg M. Natural killer T cells: natural or unnatural regulators of autoimmunity? Curr Opin Immunol. 2003;15:683-9.

24. Godfrey DI, MacDonald HR, Kronenberg M, Smyth MJ, Van Kaer L. NKT cells: what's in a name? Nat Rev Immunol. 2004;4:231-7.

25. O'Reilly V, Zeng SG, Bricard G, Atzberger A, Hogan AE, Jackson J, et al. Distinct and overlapping effector functions of expanded human $\mathrm{CD} 4+$, CD8alpha+ and CD4-CD8alpha- invariant natural killer T cells. PloS one. 2011;6:e28648.

26. Montoya CJ, Pollard D, Martinson J, Kumari K, Wasserfall C, Mulder $\mathrm{CB}$, et al. Characterization of human invariant natural killer $\mathrm{T}$ subsets in health and disease using a novel invariant natural killer $\mathrm{T}$ cell-clonotypic monoclonal antibody, 6B11. Immunology. 2007;122:1-14.

27. Nowak EC, Weaver CT, Turner H, Begum-Haque S, Becher B, Schreiner B, et al. IL-9 as a mediator of Th17-driven inflammatory disease. J Exp Med. 2009;206:1653-60.

28. Nakamura T, Schwander SK, Donnelly R, Ortega F, Togo F, Broderick G, et al. Cytokines across the night in chronic fatigue syndrome with and without fibromyalgia. Clin Vaccine Immunol. 2010;17:582-7.

29. Sumida T, Sakamoto A, Murata H, Makino Y, Takahashi H, Yoshida S, et al. Selective reduction of $\mathrm{T}$ cells bearing invariant $\mathrm{V}$ alpha $24 \mathrm{~J}$ alpha $\mathrm{Q}$ antigen receptor in patients with systemic sclerosis. J Exp Med. 1995;182:1163-8.

30. Furlan R, Bergami A, Cantarella D, Brambilla E, Taniguchi M, Dellabona P, et al. Activation of invariant NKT cells by alphaGalCer administration protects mice from MOG35-55-induced EAE: critical roles for administration route and IFN-gamma. Eur J Immunol. 2003;33:1830-8.

31. Pal E, Tabira T, Kawano T, Taniguchi M, Miyake S, Yamamura T. Costimulation-dependent modulation of experimental autoimmune encephalomyelitis by ligand stimulation of $\mathrm{V}$ alpha $14 \mathrm{NK} \mathrm{T}$ cells. J Immunol. 2001;166:662-8.

32. Huth TK, Brenu EW, Nguyen T, Hardcastle SL, Johnston S, Ramos S, et al. Characterization of Natural Killer Cell Phenotypes in Chronic Fatigue Syndrome/Myalgic Encephalomyelitis. J Clin Cell Immunol. 2014;5:1-8.

33. Wucherpfennig KW, Newcombe J, Li H, Keddy C, Cuzner ML, Hafler DA. Gamma delta T-cell receptor repertoire in acute multiple sclerosis lesions. Proc Natl Acad Sci U S A. 1992;89:4588-92.

34. Stinissen P, Zhang J, Vandevyver C, Hermans G, Raus J. Gammadelta T cell responses to activated $\mathrm{T}$ cells in multiple sclerosis patients induced by $\mathrm{T}$ cell vaccination. J Neuroimmunol. 1998;87:94-104.

35. Droogan AG, Crockard AD, Hawkins SA, McNeill TA. Gamma delta T cell distribution in cerebrospinal fluid and peripheral blood of patients with multiple sclerosis. J Neurol Sci. 1994;126:172-7.

36. Carruthers BM, van de Sande MI, De Meirleir KL, Klimas NG, Broderick G, Mitchell T, et al. Myalgic encephalomyelitis: International Consensus Criteria. J Intern Med. 2011;270:327-38.

37. Nakatomi Y, Mizuno K, Ishii A, Wada Y, Tanaka M, Tazawa S, et al Neuroinflammation in Patients with Chronic Fatigue Syndrome/ Myalgic Encephalomyelitis: An (1)(1)C-(R)-PK11195 PET Study. J Nucl Med. 2014;55:945-50

38. Wong N, Nguyen T, Brenu EW, Broadley S, Staines D, Marshall-Gradisnik S. A Comparison of Cytokine Profiles of Chronic Fatigue Syndrome/ Myalgic Encephalomyelitis and Multiple Sclerosis Patients. International Journal of Clinical Medicine. 2015;6:769. 